\title{
Knowledge and practice of contraception in United Arab Emirates women
}

Saad Ghazal-Aswad, MD, DFF, FRCOG, PhD, Associate Professor; Diaa EE Rizk, MSc, MD, FRCS, MRCOG, Assistant Professor, Department of Obstetrics and Gynaecology, Faculty of Medicine and Health Sciences, United Arab Emirates University; Samiha M. Al-Khoori, MB BS, Resident, Department of Obstetrics and Gynaecology, Al-Ain Hospital, Al-Ain, United Arab Emirates; Huda Shaheen, MSc, Research Fellow, Department of Biology, Faculty of Science, United Arab Emirates University; Letha Thomas, BSc, Research Technician, Department of Obstetrics and Gynaecology, Faculty of Medicine and Health Sciences, United Arab Emirates University.

Correspondence: Dr S Ghazal-Aswad, P.O. Box 17666, Al-Ain, United Arab Emirates. Tel: (971-3) 7672000, Fax: (971-3) 7672067, Email: saad.ghazal_Aswad@uaeu.ac.ae

(Accepted $30^{\text {th }}$ March 2001)

The Journal of Family Planning and Reproductive Health Care 2001: 27(4): 212-217

\begin{abstract}
Objective. To determine the knowledge and practice of contraception among United Arab Emirates (UAE) women. Method. Four hundred and fifty UAE women at risk of pregnancy were randomly selected from the community and primary health care centres and interviewed about knowledge and practice of contraception using a structured questionnaire.

Results. Four hundred women (89\%) gave consent to participate in the study. One hundred and sixty-six participants $(41.5 \%)$ were using contraception. All used natural methods backed with other methods. There were significant associations between using contraception and each of age, high level of education and low family income $(p<0.0001$ for the three variables). Religious beliefs and low expectation of success of birth control were the reasons given for non-use. Eighty-five percent of subjects did not accept sterilisation without medical indications, nor using contraception before the first pregnancy. Of the women, $42.5 \%$ believed that contraceptive methods should not be used after the age of 40 , and $78 \%$ were unaware that they could be used for treatment of gynaecological diseases. Disturbed bleeding patterns occurred in $48.7 \%$ of users, and these were most bothered by the inability to pray (100\%) and to have sexual intercourse (97.5\%).

Conclusion. Contraception is not commonly used by UAE women because of sociocultural traditions, religious beliefs and poor knowledge.
\end{abstract}

\section{Key message points}

- Contraception is not commonly used by UAE women because of sociocultural traditions, religious beliefs and poor knowledge.

- There were significant associations between using contraception and each of age, high level of education and low family income.

- The most common contraceptive methods used were natural methods, followed by the intra-uterine contraceptive device.

- Eighty-five percent of subjects rejected sterilisation without medical indications, and use of contraception before the first pregnancy.

- Primary health care physicians were cited as main source of contraceptive information. They therefore have a major role to play in improving the women's awareness of different contraceptive methods available and their advantages.

- Nearly half of contraceptive users reported disturbed bleeding patterns and were most bothered by the inability to pray and to have sexual intercourse.

\section{Introduction}

The introduction of modern contraceptive techniques over the past few decades and the increasing availability of safer and more effective methods of preventing pregnancy, have allowed people throughout the world to exercise their choice, make responsible decisions concerning their reproduction and enjoy the benefit of family planning. The current prevalence of contraceptive use is thus approaching $60 \%$ worldwide, and in the less developed countries approximately $53 \%$ of couples are using some form of contraception. ${ }^{1,2}$

Lack of knowledge and wrong perception about possible side effects of contraceptive methods are important reasons for non-compliance in contraceptive use and contribute to method failure and unwanted pregnancies. ${ }^{1,2}$ For example, among patients requesting abortion, misconceptions about the personal risk associated with the use of contraceptive methods and the fear of side effects were important reasons for non-use of contraception. ${ }^{3}$ Other important factors affecting the use of certain contraceptive methods are socioeconomic status, cultural background and religious belief. ${ }^{1}$ Two studies have reported on the effects of socio-economic status on knowledge and use of contraception. In adolescents in developing countries there was a significant relationship between marital status and sexual behavior, contraceptive knowledge and use. ${ }^{4}$ Among Asian women in the UK there was a significant influence of marital and professional status on contraceptive knowledge and use. However, neither of these two studies considered the contribution of cultural background nor of religious beliefs on use of contraception. Cultural and religious factors could influence the decision to use contraception before or after marriage, and before or after the first conception.

In the UAE there is high serial parity because of a deliberate pronatalist policy, and family planning is not encouraged. The cultural and religious importance of having children further limits the acceptance of birth control among the population.

The aim of the present study was to investigate the knowledge and practice of contraception of UAE women whose cultural behavior is mainly guided by dictates of their religion - Islam.

\section{Method}

A study was conducted on a randomly selected group of UAE women at risk of pregnancy, defined as married women of reproductive-age (15-44 years) who had previously born children. The main reason is that in our culture most women get married around the age of 16-18 After getting married they tend not to use contraception before they have had at least two or three children. These findings were observed in a pilot study where none of the participants used contraception before the first pregnancy and extra-marital relations were not discussed, since this is neither acceptable nor practiced in Islamic culture. The Research Ethical Committee of the Faculty of Medicine and 
Health Sciences, UAE University, Al-Ain, UAE approved the study protocol.

The survey was divided into three phases with the following objectives:

1. Pilot study: to find out the local name(s) used for different methods of birth control by UAE women and their attitude towards discussing this topic using structured interviews. This involved patients attending the Gynaecology clinic at Al-Ain Hospital (a local teaching hospital) for reasons other than contraceptive counselling.

2. Community-based qualitative survey: to determine the prevalence of contraceptive practice in a community sample of UAE women at risk of pregnancy, and to collect general background information on factors such as knowledge, taboos, and religious observances that may affect contraceptive use.

3. Primary health care-based descriptive study: to determine the prevalence of contraceptive practice in a sample of UAE women at risk of pregnancy attending the primary health care centres in UAE for medical conditions that do not include gynaecological disorders.

Sample size calculation indicated that a study of 450 subjects was adequate to achieve a high degree of precision $(95 \%)$ in estimating the true prevalence of contraceptive practice, with a priori assumption that the expected prevalence of contraceptive utilisation is $50 \%{ }^{6}$ Between January 1999 and February 1999, 450 eligible women were recruited, and of these 400 (89\%) gave consent to participate in the study. Subjects were selected using a multi-stage sampling technique. The pilot study included 50 successive patients seen at the Gynaecology clinic of Al-Ain hospital.
The community stage consisted of a random sample of 200 married mothers, aged 15-44 years, identified through the listings of the UAE women association and contacted at home. The clinic-based stage was a consecutive sample of 200 mothers, aged 15-44 years, visiting the services of the primary health care centres for non-gynaecological reasons during the study period.

Data were collected by interview administered by a trained research scientist (community-based sample) and a resident obstetrician and gynaecologist (clinic-based sample) using the structured and pre-tested questionnaire. This consisted of 23 items that included a number of demographic, reproductive and lifestyle variables (Table 1) and combined closed and open questions. Questions were generated from responses to the pilot study and modified accordingly. Care was taken to keep the questionnaire answers anonymous and confidential.

The survey definition of 'using contraception' was 'any use of a method by the couple after marriage with the aim of controlling birth'. The definition of 'current or recent user' was the use of contraception in the previous 12 months only and the 'previous user' was 'before 12 months'. Only current users were included, and previous users were classified as non-users in our study. Parity was the number of previous pregnancies $>28$ weeks.

Data were analysed using the Statistical Package for the Social Sciences (SPSS Inc., Chicago, Ill.-version 9). The following tests were employed: Student-t test and Chisquare test where appropriate, the Fisher exact test (twotailed) when the sample size was small, and the MannWhitney $U$ test for non-parametric distribution. For all analysis, a $\mathrm{p}<0.05$ was considered significant.

Table 1 Demographics of study population $(n=400)$

\begin{tabular}{|c|c|c|c|}
\hline & Users $(n=166)$ & Non-users $(n=234)$ & Difference \\
\hline Age (years) & $30.7 \pm 12.5(17-49) \dagger$ & $19.6 \pm 11.9(18-37)$ & $\mathrm{p}<0.0001$ \\
\hline Parity & $3.8 \pm 2.4(2-17)$ & $4.5 \pm 3.5(1-9)$ & NS \\
\hline \multicolumn{4}{|c|}{ Family income (Dirhams/month) } \\
\hline$<5,000$ & $16(3.6) \ddagger$ & $27(11.5)$ & $\mathrm{p}<0.01$ \\
\hline $5,000-10,000$ & $100(60.2)$ & $11(4.7)$ & $\mathrm{p}<0.0001$ \\
\hline $10,000-15,000$ & $42(25.3)$ & $106(45.2)$ & $\mathrm{p}<0.0001$ \\
\hline$>15,000$ & $8(4.8)$ & $90(38.4)$ & $\mathrm{p}<0.0001$ \\
\hline \multicolumn{4}{|l|}{ Education } \\
\hline University & $70(42.1)$ & $19(8.1)$ & $\mathrm{p}<0.0001$ \\
\hline Secondary school & $62(37.3)$ & $32(13.6)$ & $\mathrm{p}<0.0001$ \\
\hline Primary school & $23(13.8)$ & $89(38.1)$ & $\mathrm{p}<0.0001$ \\
\hline Illiterate & $11(6.6)$ & $96(41.1)$ & $\mathrm{p}<0.0001$ \\
\hline \multicolumn{4}{|l|}{ Occupation } \\
\hline Housewife & $101(60.8)$ & $153(65.3)$ & NS \\
\hline Professional & $52(31.3)$ & $66(28.2)$ & \\
\hline Manual & $13(7.8)$ & $15(6.4)$ & \\
\hline Smoking habits & $6(3.6)$ & $7(2.9)$ & NS \\
\hline \multicolumn{4}{|c|}{ Education of husband } \\
\hline University & $68(40.9)$ & $89(38.1)$ & NS \\
\hline Secondary school & $55(33.1)$ & $70(29.9)$ & \\
\hline Primary school & $23(13.8)$ & $39(16.6)$ & \\
\hline Illiterate & $20(12.1)$ & $36(15.3)$ & \\
\hline \multicolumn{4}{|c|}{ Occupation of husband } \\
\hline Professional & $121(72.8)$ & $154(65.8)$ & \\
\hline Private business & $41(24.6)$ & $71(30.3)$ & \\
\hline Manual & $3(1.8)$ & $7(2.9)$ & \\
\hline Unemployed & $1(0.6)$ & $2(0.08)$ & NS \\
\hline
\end{tabular}

$\dagger$ Mean \pm SD (Range).

$\$$ The value in parenthesis is the percentage. 


\section{Results}

A total of 400 women out of 450 enrolled subjects participated in the study, giving a non-respondent rate of $11 \%$. No further data were available on the 50 women who declined to be interviewed. Two hundred and thirty-four women $(58.5 \%)$ were not contraceptive users, 40 (14.7\%) of these had used contraception in the past but not in the previous 12 months. One hundred and sixty-six women $(41.5 \%)$ were currently using contraception. Table 1 gives the socio-demographic and reproductive characteristic of study subjects. Both users and non-users were comparable in the various parameters except for age, level of education, and family income. The association between using birth control and each of age, high level of education and low family income was highly significant $(\mathrm{p}<0.0001)$ on univariate analysis.

Figure 1 represents the methods used by UAE women in order of frequency, and these were natural methods (100\%), such as the safe period or coitus interruptus, intra-uterine contraceptive device (IUD: $56.5 \%$ ), combined oral contraceptive pill (COC: $52.4 \%$ ), progesterone-only pill (POP: 41.6\%), injectable contraceptives (16.3\%), barrier methods (condoms: 15\%) and sterilisation (6.6\%). It is important to note that Figure 1 represents different methods used by women over the last 12 months. Women could have used one or two methods previously, concomitantly or subsequently. Dual use of safe period with a barrier method was reported by $7 \%$ of the subjects.

Figure 1 Different contraceptive methods used in the study $(n=166)$. Values are given as numbers and (percentage) *.

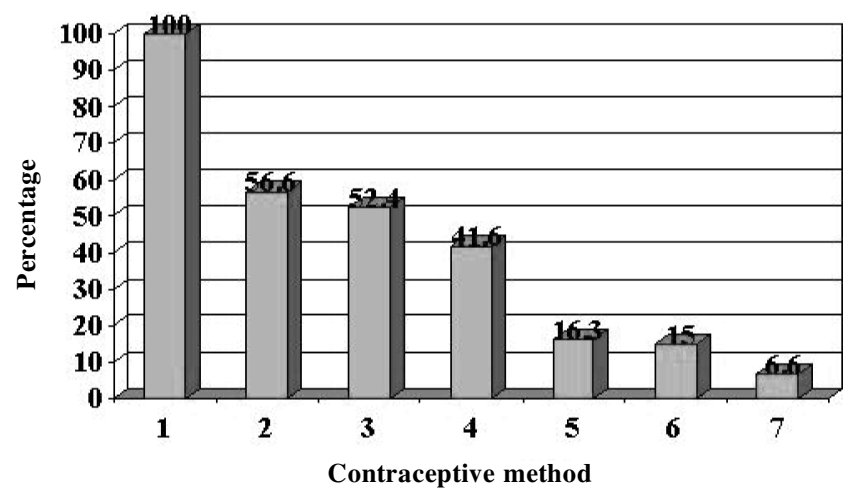

1- Natural methods, $166(100 \%)$

2- Intra-uterine contraceptive device, $94(56.5 \%)$

3- Combined oral contraceptive pill, 87 (52.4\%)

4- Progesterone-only pill, $69(41.6 \%)$

5- Injectable contraceptives, 27 (16.3\%)

6- Barrier methods, 25 (15\%)

7- Sterilisation, $11(6.6 \%)$

* Some women used more than one method

The reasons for choosing a particular contraceptive method in the user group $(n=166)$ are given in Figure 2. The important factors were recommendation of physicians and advice of friends or relatives.

The reasons given by non-users for not using contraception are listed in Table 2; $70.9 \%$ believed that using birth control was against religious teachings. This was followed by low expectation of success (33.3\%), cultural constraints imposed by relatives and friends $(23.1 \%)$ and ethical reservations on the use of contraception after marriage because the primary moral objective of marriage in the local culture is procreation (10.2\%). Only 64 non-users $(27.3 \%)$ recalled that their local primary health care physician had counselled them about contraception as part of routine health care.
Figure 2 Reasons for choosing a particular contraceptive method in users $(n=166)$. Values are given as numbers and (percentage) *.

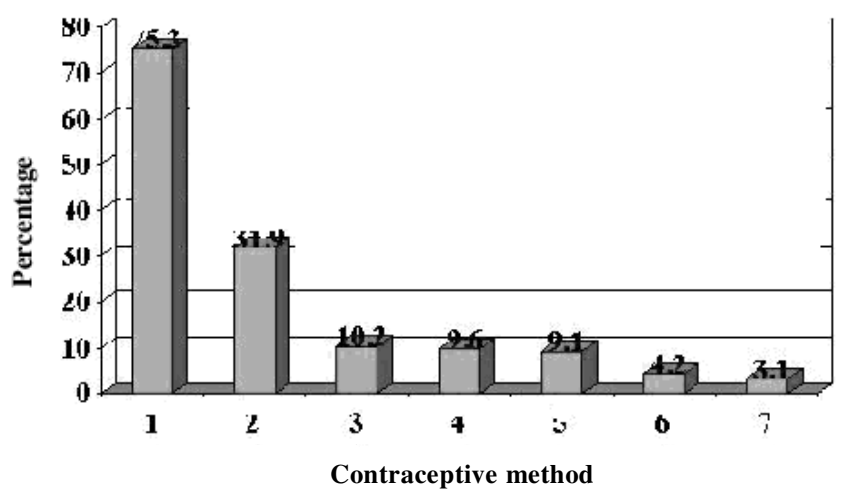

1- Recommendation of physicians 125 (75.3)

2- Advice of friends or relatives 53 (31.9)

3- Inconvenience of other methods 17 (10.2)

4- Personal choice based on information given in mass media 16 (9.6)

5- Contraindication to other methods 15 (9.1)

6- Complication of other methods 7 (4.2)

7- Failure of other methods 5 (3.1)

* Some women gave more than one answer

Table 2 Reasons given by non-users $(n=234)$ for not using contraception.*

\begin{tabular}{|c|c|}
\hline & $\begin{array}{l}\text { Non-users } \\
(\mathrm{n}=234)\end{array}$ \\
\hline Contraceptive practice is against religious teachings & $166(70.9) \dagger$ \\
\hline $\begin{array}{l}\text { The expected benefit of medical management of birth } \\
\text { control is low because most contraceptive methods fail }\end{array}$ & $78(33.3)$ \\
\hline $\begin{array}{l}\text { Embarrassed to use birth control because most of my } \\
\text { relatives and friends do not approve of birth control }\end{array}$ & $54(23.1)$ \\
\hline $\begin{array}{l}\text { Prefer not to use birth control because this is against } \\
\text { the ethical values of marriage }\end{array}$ & $24(10.2)$ \\
\hline
\end{tabular}

The perceptions of study subjects about the use of birth control are presented in Table 3. The majority accepted the idea of using contraceptive methods for the treatment of gynaecological diseases in unmarried women $(70 \%$ of users, $64 \%$ of non-users) or for birth spacing only $(87.4 \%$ of users, $86.8 \%$ of non-users). Three hundred and forty women $(85 \%)$ of the total sample, however, felt that sterilisation without a strong medical indication is against their religious $(55 \%)$, cultural $(38 \%)$ or personal $(7 \%)$ beliefs. Two hundred and ninety-six women (74\%) were reluctant to use contraception before the first pregnancy.

The majority of the respondents $(99 \%)$ were aware of the availability of birth control. Awareness of the IUD was the highest (369 subjects, $92.4 \%$ ), followed by the COC (342 subjects, $85.5 \%)$. Injectable contraceptives were the least well known (173 subjects, $43.2 \%)$. Three hundred and twelve women $(78 \%)$ were not aware that contraceptive methods could be used for treatment of some gynaecological diseases, particularly abnormal uterine bleeding. Forty-two and a half percent of women cited age 40 as the upper age limit for pregnancy risk and consequently for the need to use contraception.

Eighty-one users $(48.7 \%)$ complained of menstrual disturbances (abnormal uterine bleeding and/or dysmenorrhoea) as a result of contraceptive practice, and the consequences of these are given in Table. 4. Among users of the COC, $67.2 \%$ and $53.4 \%$, respectively, 
Use of contraceptive methods for treatment of gynaecological diseases in unmarried women

Use of contraceptive methods for birth spacing only

Sterilisation as a method of birth control without medical indications

Users agreeing

$(\mathrm{n}=166) \dagger$
Non-users agreeing $\quad \mathrm{P}$ value

$(\mathrm{n}=234) \dagger$

Use of contraceptive methods for birth control before the first pregnancy

Use of contraceptive methods for permanent birth control after completing the family instead of birth spacing only

$117(70.4) \ddagger$

$145(87.3)$

$33(19.8)$

$60(36.1)$

$21(12.6)$
$152(64.9)$

$203(86.8)$

$44(18.8)$

$\mathrm{p}<0.001$

$31(13.2)$

† Women who gave a positive answer.

\$ The value in parenthesis is the percentage.

NS $=$ Not significant

complained of headache and weight gain, while $48.1 \%$, $36.2 \%$ and $12.1 \%$, respectively, were concerned about hypertension, diabetes mellitus and return of fertility. Amongst users of the IUD, $11.9 \%$ were worried about the occurrence of pelvic inflammatory disease.

Table 4 Consequences of having menstrual disturbances in women using contraception ( $n=81,48.7 \%$ of users).*

\begin{tabular}{lc}
\hline Consequences & Number** \\
\hline $\begin{array}{l}\text { Interference with regular praying } \\
\text { Inability to have sexual intercourse }\end{array}$ & $81(100)$ \\
$\begin{array}{l}\text { Feeling disgusted and dirty about myself } \\
\text { Feeling conscious, ashamed and embarrassed especially } \\
\text { with my husband and children }\end{array}$ & $72(88.8)$ \\
$\begin{array}{l}\text { Limitation of social activities such as shopping and } \\
\text { visiting friends }\end{array}$ & $64(79.1)$ \\
$\begin{array}{l}\text { Difficulty in performing physical activities including } \\
\text { housework and job assignments }\end{array}$ & $59(72.8)$ \\
\hline
\end{tabular}

* Some women gave more than one answer

** Values are given as numbers and (percentage).

\section{Discussion}

This survey provides information not previously available from a Middle Eastern female population on the knowledge and practice of contraception. A United Nations study of global contraceptive use in $1994^{1}$ reported a prevalence of $58 \%$ in Asia and Oceania, with a range between $79 \%$ for eastern Asia compared to $43 \%$ in other Asian countries. The later is similar to the level of use in our community $(41.5 \%)$. The main reasons for non-use in the present study was that contraception was seen as contrary to religious teaching (79.9\% of subjects), was perceived as having a high chance of failure (33\% of subjects) or was believed to be culturally unacceptable (23\% of subjects). These findings are similar to those reported from similar populations. . $^{1,7}$

While natural methods are acceptable in most societies, culture and religion have great impact on use of modern contraceptives. ${ }^{7-10}$ Not surprisingly, the most common contraceptive methods used in our study were natural methods such as the safe period and coitus interruptus. The IUD was the most commonly used modern method (56.6\%) as is the case in most developing countries. ${ }^{1}$ The COC $(52.4 \%)$ and the POP $(41.6 \%)$ were the third and fourth most frequently used methods of contraception, respectively. Sterilisation was only used by $6.6 \%$ of our users for reasons cited elsewhere.

In many developed and developing countries there is an increased usage of contraception with age, ${ }^{1}$ as seen in our study. There was, however, no significant association between contraceptive usage and parity in our series, unlike in other studies ${ }^{9,10}$ since large families are still accepted and traditionally desired in the UAE. Our study confirms a significant association between use of modern methods of contraception and the educational attainment of the woman, but not her husband. ${ }^{11,12}$ This finding is important because women's education is increasing in our country and might be of value in planning the subsequent reproductive and social care of UAE women.

A significant positive association was also noted between the lower and middle range of family income and using a contraceptive method in our study. In the upper range of family income, the association became negative (i.e. $83.6 \%$ of the non-users were in the upper income group). Since the more affluent social classes were over-represented in the sample $(63 \%)$, a possible explanation could be that this group hold the traditional views of having a large family more strongly than the other classes, partly because of their privileged economic circumstances.

Similar to most series from developing countries, ${ }^{9,10}$ none of the women surveyed in our sample used, or thought about using, contraception prior to their first pregnancy. The reason could be the traditional belief that the ethical objective of marriage is procreation and accordingly contraception should only be used later on for spacing pregnancies and/or in the presence of disease.

In contrast to western experience, the main reasons for choosing a particular contraceptive method in our study were recommendation of physicians $(75.3 \%)$ or friends $(31.9 \%)$. This clearly demonstrates the importance of physicians, especially primary health care doctors, in providing the right and sound advice about contraception to women in societies like ours.

Although nearly $99 \%$ of subjects knew about contraception, the age limit was thought to be 40 years by $42.5 \%$ of women. Only $20 \%$ of users and $14 \%$ of non-users appreciated that the COC could be used to treat abnormal uterine bleeding after performing the necessary investigations to establish its cause. This observation highlights the gap between lay and biomedical knowledge of human reproduction, and of the non-contraceptive benefits of available contraceptive methods. ${ }^{7}$ Raising awareness of the efficacy of modern contraceptive methods and that these methods, especially the COC, are not harmful or unsafe requires education of more health professionals, as only $27.3 \%$ of our population had been counselled about contraception by their primary health care physician as part of routine health care. 
It is noteworthy that nearly half of the users in our study stated that they did not accept abnormal uterine bleeding because of the associated inability to pray or to have sexual intercourse. This finding stresses the importance of considering the cross-cultural differences of potential users, especially the religious traditions of Moslem women, before prescribing a contraceptive method. ${ }^{1,7}$

\section{Conclusion}

Modern methods of contraception are not widely used by UAE women, since only $41.5 \%$ used such methods. In nonusers, the main reason is the perception that birth control is against religious beliefs and social traditions. There was a significant association between using birth control and each of age, high level of education and low family income. The methods used in order of frequency were natural methods (used by nearly all respondents) followed by IUDs, COCs, POPs, injectable contraceptives and barrier methods. Sterilisation as a method of contraception was only accepted on medical grounds.

The principal reason for choosing a particular contraceptive method was recommendation of physicians.
The majority of the women were not aware that certain contraceptive methods could be used for treatment of some gynaecological diseases and that contraception should be practiced after the age of 40 years.

\section{Statements on funding and competing interests}

Funding. None.

Competing interests. None declared.

References

Levels and trends of contraceptive use as assessed in 1994. New York: United Nation document, 1996.

Huezo CM. Current reversible contraceptive methods: a global perspective. Int J Gynecol Obstet 1998; 62 (Suppl. 1): S3-S15.

Goraya A, Prakash M. Contraceptive knowledge and practice of pregnant teenagers requesting termination of pregnancy in inner city London. Fam Pract 1998; 15 (Suppl.): S14-15. Blanc AK, Way AA. Sexual behavior and contraceptive knowledge and use among adolescents in developing countries. Stud Fam Plann 1998; 2: 106-116.

Hennink M, Cooper P, Diamond I. Asian women's use of family planning services. Br J Fa Plann 1998; 24: 43-52

Cochran WG. Sampling techniques. 3rd edition, New York: John Wiley and sons 1977: 72-88. Newton J. Contraceptives: regional perspectives, issues and unmet needs - the Europea

France. Fam Plann Perspectives Adinma JIB, Agbi

Abai AO, Nwosu BO. Contraceptive choices among Nigerian women attending Contracept 1998; 14: 131-145.

Kumari C. Contraceptive practices of women living in rural areas of Bihar. Br J Fam Plan Ekani-Bessala MM, Carre N, Calvez T, et al. Prevalence and determinants of curren contraceptive method use in Palm Oil Company in Cameroon. Contraception 1998; 58: 29-34. Sihvo S, Hemminki P, Kosunen E. Contraceptive health risks - women's perceptions. Psychosom Obstet Gynecol 1998; 19: 117-125. 